\title{
Epidemiology and clinical characteristics of sarcoidosis: an update from a population-based cohort study from Olmsted County, Minnesota
}

\author{
P. Ungprasert' ${ }^{1}$, C.S. Crowson ${ }^{1,2}$, E.L. Matteson ${ }^{1,3}$ \\ 'Division of Rheumatology, Department of Internal Medicine, \\ Mayo Clinic College of Medicine and Science, Rochester, MN, USA; \\ ${ }^{2}$ Division of Biomedical Statistics and Informatics, Department of Health Science Research, \\ Mayo Clinic College of Medicine and Science, Rochester, MN, USA; \\ ${ }^{3}$ Division of Epidemiology, Department of Health Science Research, \\ Mayo Clinic College of Medicine and Science, Rochester, MN, USA
}

\begin{abstract}
SUMMARY
Information about the epidemiology, clinical manifestations and comorbidities of sarcoidosis among Caucasians is relatively scarce. This review focuses primarily on the data from a recently published Caucasianpredominant population-based cohort from Olmsted County, Minnesota. Overall, the incidence rate was 10.0 per 100,000 population, which suggested that sarcoidosis is less common in Caucasians than in Blacks, but is more common in Caucasians than in Asians. Intrathoracic involvement was seen in the vast majority of patients, but less than half have respiratory symptoms. The most common extra-thoracic manifestations were skin rash followed by arthralgia, ophthalmologic involvement, hepatic involvement, splenomegaly, renal involvement, neurological involvement, extra-thoracic lymphadenopathy, exocrine gland involvement, upper respiratory tract involvement and cardiac involvement. Compared to sex and age-matched subjects, patients with sarcoidosis suffer from increased rates of cardiovascular disease, venous thromboembolism and hospitalized infection.
\end{abstract}

Key words: Sarcoidosis; pulmonology; interstitial lung disease.

Reumatismo, 2017; 69 (1): 16-22

\section{INTRODUCTION}

Sarcoidosis is a multi-system disorder of unclear etiology characterized by the presence of non-caseating granuloma, which are comprised of macrophages, epithelioid cells, mononuclear cells and CD4+ T cells with a few $\mathrm{CD} 8+\mathrm{T}$ cells in the peripheral zone. Lung and intra-thoracic lymph node are the most commonly affected organs although any organs can be involved. The clinical course of sarcoidosis ranges from an indolent process to an acute self-limited process to a progressive disease with permanent organ damage $(1,2)$.

Patompong Ungprase Division of Rheumatology, Mayo Clinic, 200 First avenue SW,

Rochester, MN 55905, USA

E-mail: p.ungpraser@@gmail.com ungprasert.patompong@mayo.edu
There is only limited information regarding the epidemiology and clinical characteristics and outcome of sarcoidosis, partic- ularly among Caucasians. The purpose of this review is to summarize the data from a recently published Caucasian-predominant cohort of residents of Olmsted County, Minnesota, United States who were first diagnosed with sarcoidosis between 1976 and 2013 with an emphasis on epidemiology, clinical characteristics and comorbidity (3).

\section{EPIDEMIOLOGY OF SARCOIDOSIS}

\section{Incidence and mortality}

In this population, the annual incidence of sarcoidosis among adults in 1946-2013 was 10.0 per 100,000 population. Sarcoidosis was slightly more common among fe- 
males than males $(10.5$ per 100,000 population among females and 9.4 per 100,000 population among males). No significant calendar year trend in incidence was observed in either females or males. Mean age at diagnosis was 48.3-years in females and 42.8 years in males. The observed incidence rate was in line with other Caucasian studies that reported incidence rates of 5 to 19 per 100,000 population per year (4-6) and prevalence of 160 per 100,000 population (6). Compared to other ethnic groups, the incidence rate of sarcoidosis among Caucasians is lower than Blacks (with reported incidence of 40 to 70 per 100,000 population per year) $(7,8)$ but is higher than Asians (with reported incidence of 1 per 100,000 population per year) (9).

The overall mortality of patients with sarcoidosis in this cohort was not different from the general population. This is in contrast to previous reports that observed an approximately 2 -fold increased rate of mortality $(4,10)$. This discrepancy may reflect differences in management of sarcoidosis in different regions or the difference of severity and outcome of sarcoidosis across ethnic groups $(11,12)$.

\section{Risk factors}

Despite decade-long research effort, the etiology and pathogenesis of sarcoidosis remains poorly understood. A widely accepted hypothesis is that of a complex interaction between environmental factors and genetic factors (13). Data from this U.S. population-based cohort may provide further insights into the possible etiologies of sarcoidosis.

First, there is some seasonality of sarcoidosis, as the incidence rate of sarcoidosis was persistently lower in autumn compared to the rest of the year across 4 decades of study (14). This may reflect seasonality of environmental trigger(s) associated with the granuloma-formation in sarcoidosis. Nonetheless, this observed seasonal variation is not consistent across different regions of the world. For example, a study from Turkey (15) reported the lowest incidence in summer, while a study from India (16) observed the lowest incidence in win- ter. The different patterns of seasonality might reflect different environmental stimuli. Second, obesity was associated with a higher risk of sarcoidosis. The odds of developing sarcoidosis were about 2.4 times higher among obese subjects (i.e., body mass index $\geq 30 \mathrm{~kg} / \mathrm{m}^{2}$ ) compared to those who were not obese (17). While certainly speculative, this observation may suggest the role of leptin, a hormone secreted by adipocytes, in the pathogenesis of sarcoidosis. Leptin is a pro-inflammatory adipokine with a potent immuno-modulatory effect that is able to sustain autoreactive cell proliferation, which may increase the risk of autoimmune disease, including sarcoidosis (18). An increased risk of autoimmune diseases, such as psoriasis and rheumatoid arthritis, has been described among obese subjects (19).

Third, the risk of developing sarcoidosis was lower among active smokers. The odds ratio of sarcoidosis comparing current smokers with never smokers and former smokers adjusted for age and sex was 0.38 (17). This is an interesting observation, as smoking is generally associated with a higher risk of pulmonary disease, such as chronic obstructive pulmonary disease and lung cancer. The lower risk of sarcoidosis among active smokers has been demonstrated in other cohorts as well $(20,21)$. Furthermore, smoking has been associated with severity mitigation in another autoimmune disease, ulcerative colitis (22). It is known that smoking can suppress T-lymphocyte function and phagocytic activity of macrophages. Possibly of relevance to sarcoidosis, it is also possible that smoking might interfere with the macrophagelymphocyte interaction that results in the formation of granuloma (23).

\section{CLINICAL MANIFESTATIONS}

\section{Pulmonary sarcoidosis}

Intrathoracic involvement was the characteristic finding among patients with sarcoidosis that was seen in the vast majority of the cohort $(97 \%)$. In $87 \%$ of cases this consisted of intrathoracic lymphadenopathy, and $50 \%$ had evidence of pulmonary 
parenchymal infiltration. The higher frequency of intrathoracic lymphadenopathy compared with pulmonary parenchymal infiltration is expected as patients with stage I pulmonary sarcoidosis have only intrathoracic lymphadenopathy without any pulmonary parenchymal abnormality. The relatively low sensitivity of conventional chest radiography in detecting infiltration in the lung parenchyma may also contribute to this discrepancy as only about $50 \%$ of patients in this cohort had computed tomography scan of the lung.

However, respiratory symptoms were relatively infrequent, and occurred in less than half of the patients (43\%) (3). This observation suggests the necessity of thoracic imaging, preferably computed tomography scanning, if sarcoidosis is suspected, despite lack of pulmonary symptoms.

\section{Extra-thoracic sarcoidosis}

Extra-thoracic manifestations were observed frequently in this cohort (3). The most common extra-thoracic manifestations were skin rash (18\%), followed by arthralgia (12\%), ophthalmologic involvement $(7 \%)$, hepatic involvement $(6 \%)$, splenomegaly (4\%), renal involvement (3\%), neurological involvement (3\%), extra-thoracic lymphadenopathy (3\%), exocrine gland involvement (2\%), upper respiratory tract involvement $(2 \%)$ and cardiac involvement (1\%). Bone involvement was

Table I - Frequency of organ involvement by sarcoidosis.

\begin{tabular}{|l|c|}
\hline Organ & Frequency \\
\hline Intrathoracic & $97 \%$ \\
Hilar and mediastinal lymph nodes & $87 \%$ \\
Lung parenchyma & $50 \%$ \\
\hline Skin & $18 \%$ \\
\hline Joints & $12 \%$ \\
\hline Eyes & $7 \%$ \\
\hline Liver & $6 \%$ \\
\hline Spleen & $4 \%$ \\
\hline Kidneys & $3 \%$ \\
\hline Nervous system & $3 \%$ \\
\hline Extrathoracic lymph node & $3 \%$ \\
\hline Upper respiratory tract & $2 \%$ \\
\hline Heart & $1 \%$ \\
\hline
\end{tabular}

observed in only one patient. The frequency of organ involvement by sarcoidosis is summarized in Table I.

\section{Skin}

Cutaneous involvement was the most common extra-thoracic manifestation of sarcoidosis, occurring in $18 \%$ of patients with sarcoidosis. Approximately $60 \%$ of patients with cutaneous sarcoidosis had sarcoidosis-specific skin lesions (defined as the presence of non-necrotizing granuloma on skin biopsy) while the rest had erythema nodosum (24).

Among those with sarcoidosis-specific skin lesions, subcutaneous nodules $(33 \%)$ were the most common type of lesions followed by papules $(31 \%)$, plaques $(19 \%)$, tattoo/ scar sarcoidosis $(11 \%)$, lupus pernio $(11 \%)$ and ulcer $(8 \%)$. The lesions were more frequently found on the extremities and head/ neck area than on the trunk.

Response to treatment was favorable with complete response by 2-years after diagnosis in over $80 \%$ of patients with sarcoidosis-specific skin lesions and almost all patients with erythema nodosum. The median time from the first appearance of cutaneous lesions to resolution of all skin lesions was 4.4 months, and to resolution of erythema nodosum was 1.6 months. Oral glucocorticoids were the most frequently used medication to treat patients with sarcoidosisspecific skin lesions followed by topical glucocorticoids, intralesional glucocorticoids and topical calcineurin inhibitor.

\section{Joints}

Arthropathy was the second most common extra-thoracic disease manifestation of sarcoidosis in this cohort, with joint pain occurring in $12 \%$ and swollen joints occurring in $10 \%$ of patients with sarcoidosis (25). Oligoarthritis was by far the most common pattern of joint involvement $(88 \%)$. The most commonly affected joints were ankles (91\% total with $14 \%$ one ankle and $77 \%$ both ankles), followed by knees $(29 \%)$ and wrists $(14 \%)$. Involvement of small joints and tendinitis/enthesitis was uncommon. Costochondritis, axial joint involvement and sacroiliac joint involvement 
Table II - Cumulative incidence at 10 years of selected comorbidities of patients with sarcoidosis and hazard ratio compared to sex- and age-matched subjects.

\begin{tabular}{|l|c|c|}
\hline Comorbidities & $\begin{array}{c}\text { Cumulative incidence at } 10 \text { years } \\
(95 \% \mathrm{CI})\end{array}$ & $\begin{array}{c}\text { HR }(95 \% \text { Cl) for all events } \\
\text { after index date, adjusting for age, } \\
\text { sex and calendar year }\end{array}$ \\
\hline Cardiovascular disease & $17.6(12.9-22.0)$ & $1.57(1.15-2.16)$ \\
\hline Venous thromboembolism & $4.0(1.6-6.3)$ & $3.04(1.47-6.29)$ \\
\hline Malignancy & $3.8(1.5-6.1)$ & $0.72(0.49-1.06)$ \\
\hline Hospitalized infection & $13.2(9.1-17.0)$ & $2.00(1.41-2.84)$ \\
\hline
\end{tabular}

$\mathrm{HR}$, hazard ratio; $\mathrm{Cl}$, confidence interval.

were not seen in this Olmsted County cohort. Classic sarcoidal bone lesions, such as bone cyst or lytic appearance, were not observed on radiographic investigations either. Classic Lofgren syndrome (arthritis, erythema nodosum and hilar adenopathy) was observed in about a quarter of those with joint involvement. The outcome of sarcoid arthropathy is good, as the majority of patients $(88 \%)$ achieved complete remission within 6 weeks. Most patients with active joint disease were treated with a short duration of non-steroidal anti-inflammatory drugs (NSAIDs) and/or oral glucocorticoids (25).

\section{Parotid glands}

Parotid gland involvement is a well-recognized extra-thoracic feature of sarcoidosis. However, only $2 \%$ of patients with sarcoidosis in this population-based cohort had parotid gland involvement (26). Unilateral painless gland swelling was the most common presentation of parotid gland disease. This differed from what has been reported in other cohorts, which report bilateral involvement in more than $70 \%$ of cases (27, 28 ). More importantly, parotid gland disease was the initial manifestation in 57\% of patients in the Olmsted County cohort, highlighting the vital role of the otolaryngologic assessment in the diagnosis of systemic sarcoidosis.

\section{COMORBIDITY}

Over the past few decades, morbidity and mortality from direct organ damage have been declining in patients with autoimmune diseases because of the availability of novel effective treatments, such as disease-modifying anti-rheumatic agents and biologic agents. As a result, patients with autoimmune diseases live longer, and comorbidities have emerged as a major cause of morbidity and mortality. Several studies have described the characteristics and outcomes of comorbidities in several autoimmune diseases, particularly rheumatoid arthritis and systemic lupus erythematosus $(29,30)$. However, there is a dearth of studies on comorbidities of sarcoidosis. Table II summarizes the cumulative incidence at 10-years of comorbidities examined in the Olmsted County cohort of patients with sarcoidosis and sex- and age-matched comparator subjects.

\section{Cardiovascular disease}

The risk of cardiovascular disease (CVD), including coronary artery disease (CAD), congestive heart failure (CHF), atrial fibrillation $(\mathrm{AF})$, cerebrovascular accident (CVA), transient ischemic attack (TIA), peripheral arterial disease (PAD) and abdominal aortic aneurysm (AAA), was investigated in this Olmsted County cohort using sex- and agematched subjects from the same underlying population as comparators. The cumulative incidence of all CVD events among patients with sarcoidosis at 10 years was $17.6 \%$. The risk of incident CVD, adjusted for age, sex, calendar year, was significantly higher among patients with sarcoidosis with a hazard ratio (HR) of 1.57 (95\% confidence [CI], 1.15-2.16). Further adjustment for baseline CVD risk factors yielded a similar result. Significantly elevated risk was observed for several subtypes of CVD including CAD, CHF, AF and CVA (31). 
Chronic inflammation is probably the key driver behind this increased risk of CVD (32). While not specifically studied in sarcoidosis, it has been demonstrated that several inflammatory cytokines, such as interleukin-1 and tumor necrosis factor, can promote premature atherosclerosis through a number of mechanisms including vascular adhesion molecule-1 and leukocyte and matrix metalloproteinase activation (33, 34). Chronic inflammation may trigger the initiation and propagation of the coagulation cascade, downregulate anti-coagulation pathways and inhibit removal of fibrin $(35,36)$, thereby promoting thrombosis in coronary and intra-cerebral arteries.

Glucocorticoids, the most commonly used medication for the treatment of sarcoidosis, may also be a contributing factor to the increased risk of CVD, as use of glucocorticoids is associated with several metabolic sequelae such as diabetes mellitus, hypertension and dyslipidemia, which are traditional risk factors for CVD (37).

\section{Venous thromboembolism}

Similar to CVD, an increased incidence of venous thromboembolism (VTE), including deep vein thrombosis (DVT) and pulmonary embolism (PE), has been observed in several autoimmune diseases (38-40). However, data on the risk of VTE among patients with sarcoidosis are limited. In this cohort, the cumulative incidence of VTE among patients with sarcoidosis at 10 years was $4.0 \%$, which corresponded to an approximately 3-fold increased risk of incident VTE adjusted for age, sex and calendar year. Significantly elevated risk was observed in both DVT and PE (41).

Three main predisposing factors of VTE, known as Virchow's triad, include venous stasis, endothelial injury and hypercoagulability. Chronic inflammation is known to provoke a hypercoagulable state through several mechanisms including stimulation of the coagulation cascade by increased expression of tissue factor induced by inflammatory cytokines, mobilization of clot-promoting membrane phospholipids by complement complexes and suppression of natural anti-coagulants such as thrombomodulin by tumor necrosis factoralpha $(35,36)$. Chronic inflammation can also induce endothelial injury as a result of the detrimental effect of inflammatory cytokines and oxidative stress (32). Patients with sarcoidosis may also be less active than sex- and age-matched subjects due to respiratory symptoms. These factors could predispose patients with sarcoidosis to the development of DVT and PE. In addition, use of glucocorticoids may also increase the propensity to blood clots. A case-control study has demonstrated a dose-response relationship between glucocorticoids and incidence of VTE (42).

\section{Malignancy}

The possible relationship between malignancy and sarcoidosis is a subject of debate. The possible link has been a matter of concern for decades, with several case series reporting patients diagnosed with cancer shortly before or after the diagnosis of sarcoidosis (43). Nonetheless, subsequent epidemiologic studies have yielded conflicting results (44-46).

This cohort was utilized to investigate this controversial association. There was no difference in the prevalence of malignancy at index date among patients with sarcoidosis compared to non-sarcoidosis subjects. Similarly, there was no difference in the incidence of malignancy after index date, with the cumulative incidences at 10-years of $3.8 \%$ and $7.1 \%$ for patients with sarcoidosis and comparators, respectively. The difference corresponded to HR of 0.72 (95\% CI, 0.49-1.06) (47).

\section{Infection}

Infection is one of the most common complications of autoimmune diseases. An increased risk of both pyogenic and opportunistic infection has been observed in them. Use of immunosuppressive agents and immune dysregulation related to the pathogenesis of autoimmune diseases are thought to be key drivers behind the increased risk (48).

Increased risk of hospitalized infection has been demonstrated in several autoimmune diseases (49-51). However, there had been 
no study of hospitalized infection among patients with sarcoidosis. This cohort was utilized to fill this knowledge gap. The cumulative incidence of all hospitalized infection among patients with sarcoidosis at 10 years was $13.2 \%$. The risk was increased by $200 \%$ compared to sex- and age-matched subjects. This excess risk is seen in varying degrees for all subtypes of infections examined. Use of oral glucocorticoids was a significant predictor of hospitalized infection in a dose-dependent fashion.

\section{CONCLUSIONS}

In conclusion, this review summarizes all new data from a recently published cohort of patients with sarcoidosis from Olmsted County, Minnesota. It is a relatively rare disease with manifold clinical manifestations. Comorbidities, especially risk of cardiovascular disease, are frequent.

Conflict of interest: the authors have no potential conflicts of interest to declare.

\section{REFERENCES}

1. Thomas KW, Hunninghake GW. Sarcoidosis. JAMA. 2003; 289: 3300-3.

2. Chen ES, Moller DR. Sarcoidosis - scientific progress and clinical challenges. Nat Rev Rheumatol. 2011; 7: 457-67.

3. Ungprasert P, Carmona EM, Utz JP, et al. Epidemiology of sarcoidosis 1946-2013: A populationbased study. Mayo Clin Proc. 2016; 91: 183-8.

4. Gribbin J, Hubbard RB, Jeune IL, et al. Incidence and mortality of idiopathic pulmonary fibrosis and sarcoidosis in the UK. Thorax. 2006; 61: 980-5.

5. Hillerdal G, Nou E, Osterman K, et al. Sarcoidosis: epidemiology and prognosis. A 15year European study. Ann Rev Respir Dis. 1984; 130: 29-32.

6. Arkema EV, Grunewald J, Kullberg S, et al. Sarcoidosis incidence and prevalence: a nationwide register-based assessment in Sweden. Eur Resp J. 2016; 48: 1690-99.

7. Rybicki BA, Major M, Popovich J Jr, et al. Racial differences in sarcoidosis incidence: a 5-year study in a health maintenance organization. Am J Epidemiol. 1997; 145: 234-41.

8. Cozier YC, Berman JS, Palmer JR, et al. Sarcoidosis in black women in the United States: Data from the black women's health study. Chest. 2011; 139: 144-50.
9. Morimoto T, Azuma A, Abe S, et al. Epidemiology of sarcoidosis in Japan. Eur Resp J. 2008; 31: 372-9.

10. Tukey MH, Berman JS, Boggs DA, et al. Mortality among African American women with sarcoidosis: data from the Black Women's Health Study. Sarcoidosis Vasc Diffuse Lung Dis. 2013; 30: 128-33.

11. Mirsaedi M, Machado RF, Schraufnagel D, et al. Racial difference in sarcoidosis mortality in the United States. Chest. 2015; 147: 438-49.

12. Swigris JJ, Olson AL, Huie TJ, et al. Sarcoidosis-related mortality in the United States from 1988 to 2007. Am J Respir Crit Care Med. 2011; 183: 1524-30.

13. Chen ES, Moller DR. Etiology of sarcoidosis. Clin Chest Med 2008; 29: 365-77.

14. Ungprasert P, Crowson CS, Matteson EL. Seasonal variation in incidence of sarcoidosis: a population-based study, 1976-2013. Thorax. 2016 Aug 18 [Epub ahead of print].

15. Demirkok SS, Basaranoglu M, Coker E, et al. Seasonality of the onset of symptoms, tuberculin test anergy and Kveim positive reaction in a large cohort of patients with sarcoidosis. Respirology. 2007; 12: 591-3.

16. Gupta D, Agarwal R, Aggarwal AN. Seasonality of sarcoidosis: the "heat" is on... Sarcoidosis Vasc Diffuse Lung Dis. 2013; 30: 241-3.

17. Ungprasert P, Crowson CS, Matteson EL. Smoking, obesity and risk of sarcoidosis: a population-based nested case-control study. Respir Med. 2016; 120: 87-90.

18. Procaccini C, Jirillo E, Matarese G. Leptin as an immunomodulator. Mol Aspect Med. 2012; 33: 35-45.

19. Versini M, Jeandel PY, Rosenthal E, et al. Obesity in autoimmune diseases: not a passive bystander. Autoimmun Rev. 2014; 13: 9811000.

20. Newman LS, Rose CS, Bresnitz EA, et al. A case control etiologic study of sarcoidosis: environmental and occupational risk factors. Am J Respir Crit Care Med. 2004; 170: 1324-30.

21. Valeyre D, Soler P, Clerici C, et al. Smoking and pulmonary sarcoidosis: effect of cigarette smoking on prevalence, clinical manifestations, alveolitis, and evolution of the disease. Thorax. 1988; 43: 516-24.

22. Silverstein MD, Lashner BA, Hanauer SB. Cigarette smoking and ulcerative colitis: a case-control study. Mayo Clin Proc. 1994; 69: 425-9.

23. Moller W, Bath W, Pohlit W, et al. Smoking impairs alveolar macrophage activation after inert dust exposure. Toxicol Lett. 1996; 88: 131-7.

24. Ungprasert P, Wetter DA, Crowson CS, et al. Epidemiology of cutaneous sarcoidosis, 19762013: a population-based study from Olmsted County, Minnesota. J Eur Acad Dermatol Venereol. 2016; 30: 1799-804. 
25. Ungprasert P, Crowson CS, Matteson EL. Clinical characteristics of sarcoid arthropathy: a population-based study. Arthritis Care Res (Hoboken). 2016; 68: 695-9.

26. Ungprasert P, Crowson CS, Matteson EL. Clinical characteristics of parotid gland sarcoidosis: a population-based study. JAMA Otolaryngol Head Neck Surg. 2016; 142: 503-4.

27. James DG, Sharma OP. Parotid gland sarcoidosis. Sarcoid Vasc Diffuse Lung Dis. 2000; 17 : 27-32.

28. Hammer JE III, Scofield HH. Cervical adenopathy and parotid gland due to sarcoidosis: a study of 31 cases. J Am Dent Assoc. 1967; 74: 1224-30.

29. Crowson CS, Liao KP, Davis JM 3rd, et al. Rheumatoid arthritis and cardiovascular disease. Am Heart J. 2013; 166: 622-8.

30. Lee YH, Choi SJ, Ji JD, et al. Overall and cause-specific mortality in systemic lupus erythematosus: an update meta-analysis. Lupus. 2016; 25: 727-34.

31. Ungprasert P, Crowson CS, Matteson EL. Risk of cardiovascular disease among patients with sarcoidosis: a population-based retrospective cohort study, 1976-2013. Eur Respir J. 2017; 49: 1601290.

32. Montecucco F, Mach F. Common inflammatory mediators orchestrate pathophysiological processes in rheumatoid arthritis and atherosclerosis. Rheumatology (Oxford). 2009; 48: 11-22.

33. Cybulsky MI, Iiyama K, Li H, et al. A major role for VCAM-1, but not ICAM-1, in early atherosclerosis. J Clin Invest. 2001; 107: 1255-62.

34. Rajavashisth TB, Liao JK, Galis ZS, et al. Inflammatory cytokines and oxidized low density lipoproteins increase endothelial cell expression of membrane type 1-matrix metalloproteinase. J Biol Chem. 1999; 274: 11924-9.

35. Xu J, Lupu F, Esmon CT. Inflammation, innate immunity and blood coagulation. Hamostaseologie. 2010; 30: 5-6, 8-9.

36. Riva N, Donadini MP, Ageno W. Epidemiology and pathophysiology of venous thromboembolism: similarities with atherothrombosis and the role of inflammation. Thromb Haemost. 2015; 113: 1176-83.

37. Maradit Kremers H, Reinalda MS, Crowson $\mathrm{CS}$, et al. Glucocorticoids and cardiovascular and cerebrovascular events in polymyalgia rheumatica. Arthritis Rheum. 2007; 57: 279-86.

38. Ungprasert P, Srivali N, Spanuchart I, et al. Risk of venous thromboembolism in patients with rheumatoid arthritis: a systematic review and meta-analysis. Clin Rheumatol. 2014; 33 : 297-304.

39. Tomasson G, Monach PA, Merkel PA. Thromboembolic disease in vasculitis. Curr Opin Rheumatol. 2009; 21: 41-6.

40. Zoller B, Li X, Sunquist J, Sunquist K. Risk of pulmonary embolism in patients with autoimmune disorders: a nationwide follow-up study from Sweden. Lancet. 2012; 379: 244-9.

41. Ungprasert P, Crowson CS, Matteson EL. Association of sarcoidosis with increased risk of venous thromboembolism: a population-based study 1976-2013. Chest. 2016; 16: 59247-2.

42. Johannesdottir SA, Horvath-Puho E, Dekkers $\mathrm{OM}$, et al. Use of glucocorticoids and risk of venous thromboembolism: a nationwide population-based case-control study. JAMA Intern Med. 2013; 173: 743-52.

43. Suen JS, Forse MS, Hyland RH, et al. The malignancy-sarcoidosis syndrome. Chest. 1990; 96: $1300-1$.

44. Askling J, Grunewald J, Eklund A, et al. Increased risk of cancer following sarcoidosis. Am J Respir Crit Care Med. 1999; 160: 166872.

45. Ji J, Shu X, Li X, Sundquist K, et al. Cancer risk in hospitalized sarcoidosis patients: a follow-up study in Sweden. Ann Oncol. 2009; 20: 1121-6.

46. Ungprasert P, Srivali N, Wijarnpreecha K, et al. Is the incidence of malignancy increased in patients with sarcoidosis? A systematic review and meta-analysis. Respirology. 2014; 19: 993-8.

47. Ungprasert P, Crowson CS, Matteson EL. Risk of malignancy among patients with sarcoidosis: A population-based cohort study. Arthritis Care Res (Hoboken). 2017; 69: 46-50.

48. Migita K, Sasaki Y, Ishizuka N, et al. Glucocorticoid therapy and the risk of infection in patients with newly diagnosed autoimmune disease. Medicine (Baltimore). 2013; 92: 285-93.

49. Doran MF, Crowson CS, Pond GR, et al. Frequency of infection in patients with rheumatoid arthritis: a population-based study. Arthritis Rheum. 2002; 46: 2281-93.

50. Herrinton LJ, Liu L, Goldfien R, et al. Risk of serious infection for patients with systemic lupus erythematosus starting glucocorticoids with or without antimalarials. J Rheum. 2007; 56: 2092-4.

51. Marie I, Menard JF, Hachulla E, et al. Infectious complications in polymyositis and dermatomyositis: A series of 279 patients. Semin Arthritis Rheum. 2011; 41: 48-60. 